J. Venom. Anim. Toxins incl. Trop. Dis.

V.11, n.2, p.101, 2005.

Editor's viewpoint - ISSN 1678-9199

\title{
MEDICAL CARE USING TELEMEDICINE TOOLS
}

The use of Telemedicine is being largely diffused with the aim of shortening the distance between health professionals and their patients.

CEVAP - Center for the Study of Venoms and Venomous Animals, UNESP - São Paulo State University, Botucatu, São Paulo, Brazil, launched in 2005 an innovative home care service in the area of accidents by venomous animals, named TelehomE-care.

It is an online system that provides help to the general population and health professionals. For this purpose, we set up an electronic medium, a way to recognize venomous animals, a bank of frequently asked questions (FAQ), and a virtual outpatient's department providing real time health care by means of videos, voice, and/or chat. The users can contact experts to solve doubts or even receive information about their diagnosis.

TelehomE-care makes possible the identification of a venomous animal in real time, the discussion of clinical cases, the monitoring of the treatment side effects, and the longdistance follow-up of a patient. This system permits medical care to the patients and communication between doctors, which may be isolated due to physical or geographical limitations.

The service can be accessed from any computer connected to the Internet, facilitating the access by the less favored communities of the country, spreading information and helping the population in a better way.

This is a model that other excellence centers could adopt in partnership with CEVAP. The proposal may be used in other knowledge areas, in which, after the diagnosis and establishment of the treatment, face-to-face meetings are not necessary to the patient follow-up.

The service is free, opened to the population and can be accessed at http://www.cevap.unesp.br/ecare.

Rui Seabra Ferreira Junior, DVM, MS, Email rseabra@cevap.org.br

Ana Silvia Sartori Barraviera S. Ferreira, Advertising, MS

Telemedicine graduate student at São Paulo University, Email anasilvia@cevap.org.br 